\title{
Using satellites to uncover large methane emissions from landfills
}

\author{
Joannes D. Maasakkers ${ }^{1, *}$, Daniel J. Varon ${ }^{2,3}$, Aldís Elfarsdóttir ${ }^{1,}{ }^{\dagger}$, \\ Jason McKeever ${ }^{3}$, Dylan Jervis ${ }^{3}$, Gourav Mahapatra ${ }^{1}$, Sudhanshu Pandey ${ }^{1}$, \\ Alba Lorente ${ }^{1}$, Tobias Borsdorff ${ }^{1}$, Lodewijck R. Foorthuis ${ }^{1}$, Berend J. Schuit ${ }^{1}$, \\ Paul Tol ${ }^{1}$, Tim A. van Kempen ${ }^{1}$, Richard van Hees ${ }^{1}$, Ilse Aben ${ }^{1}$ \\ ${ }^{1}$ SRON Netherlands Institute for Space Research, Leiden, The Netherlands \\ ${ }^{2}$ Harvard University, Cambridge, MA, USA \\ ${ }^{3}$ GHGSat, Inc., Montréal, Quebec, Canada \\ ${ }^{\dagger}$ Now at: Stanford University, Stanford, CA, USA \\ *E-mail: j.d.maasakkers@ sron.nl.
}

This is a non-peer reviewed preprint submitted to EarthArXiv 
As atmospheric methane concentrations increase at record pace, it is critical to identify individual emission sources with high potential for mitigation. Landfills are responsible for large methane emissions that can be readily abated but have been sparsely observed. Here we leverage the synergy between satellite instruments with different spatiotemporal coverage and resolution to detect and quantify emissions from individual landfill facilities. We use the global surveying Tropospheric Monitoring Instrument (TROPOMI) to identify large emission hot spots, and then zoom in with high-resolution target-mode observations from the GHGSat instrument suite to identify the responsible facilities and characterize their emissions. Using this 'tip and cue' approach, we detect and analyze strongly emitting landfills $\left(3-29 \mathbf{t ~ h r}^{-1}\right)$ in Buenos Aires (Argentina), Delhi (India), Lahore (Pakistan), and Mumbai (India). We find that city-level emissions are 1.6-2.8 times larger than reported in commonly used emission inventories and that the landfills contribute 5-47\% of those emissions. Our work demonstrates how complementary satellites enable global detection, identification, and monitoring of methane super-emitters at the facility-level.

Reducing methane emissions is a priority for curbing climate change (1-4). With global methane concentrations increasing at record pace (5), identifying sources with high potential for mitigation is a crucial first step. A small number of anomalously strong point sources ("superemitters") make up a disproportionately large fraction of total emissions and can often be readily mitigated $(3,6,7)$. Satellites have the ability to observe atmospheric methane concentrations around the world. They can be used to detect and quantify strong point sources and characterize emissions at regional and national scales for comparison with reported emissions (8). Here we leverage synergies between satellite instruments with disparate spatial resolution and coverage to detect strong urban methane hotspots, identify major sources responsible for the hotspots, 
and characterize their facility-level emissions.

Emissions from the oil and gas sector have received considerable attention (9-15), but there are also major opportunities for emission mitigation in the waste sector which accounts for roughly $12 \%$ of global anthropogenic emissions (16). Solid waste emissions are caused by the anaerobic decay of organic material in landfills. The largest historic methane emission reductions in any source sector reported to the United Nations Framework on Climate Change (UNFCCC) by Annex-I countries have been related to landfills. Reported solid waste emissions in the United States fell by 38\% between 1990 and 2018, and emissions in the European Union were nearly halved over the same time period (17). Yet landfilled waste is expected to grow at more than double the rate of population growth between now and 2050, mainly driven by countries in the tropics (18). As a result, global solid waste emissions could nearly double to $60 \mathrm{Tg} \mathrm{a}^{-1}$ by 2050 (19). Conversely, these emissions could be reduced to $11 \mathrm{Tg} \mathrm{a}^{-1}$ using technically feasible reduction strategies including active landfill covers, energy recovery, and omitting organic waste from landfills $(19,20)$. In this study we employ a multi-satellite observing framework to identify, characterize, and monitor four high-emitting landfills across the globe, including the ability to track emission mitigation measures.

Launched in October 2017, the Tropospheric Monitoring Instrument (TROPOMI) on the Sentinel-5P satellite provides daily global coverage of atmospheric methane concentrations at a spatial resolution of up to $5.5 \times 7 \mathrm{~km}^{2}(21,22)$. These data can be used to detect and quantify large emission events $(11,12)$ and regional emissions $(14,15)$, but the spatial resolution is insufficient to unambiguously pinpoint emissions from all but the strongest and most isolated methane point sources. Meanwhile, target-mode instruments like GHGSat-D, -C1, and -C2 $(23,24)$ only observe limited spatial domains $\left(\sim 10 \times 10 \mathrm{~km}^{2}\right)$ but do so at fine pixel reso- 


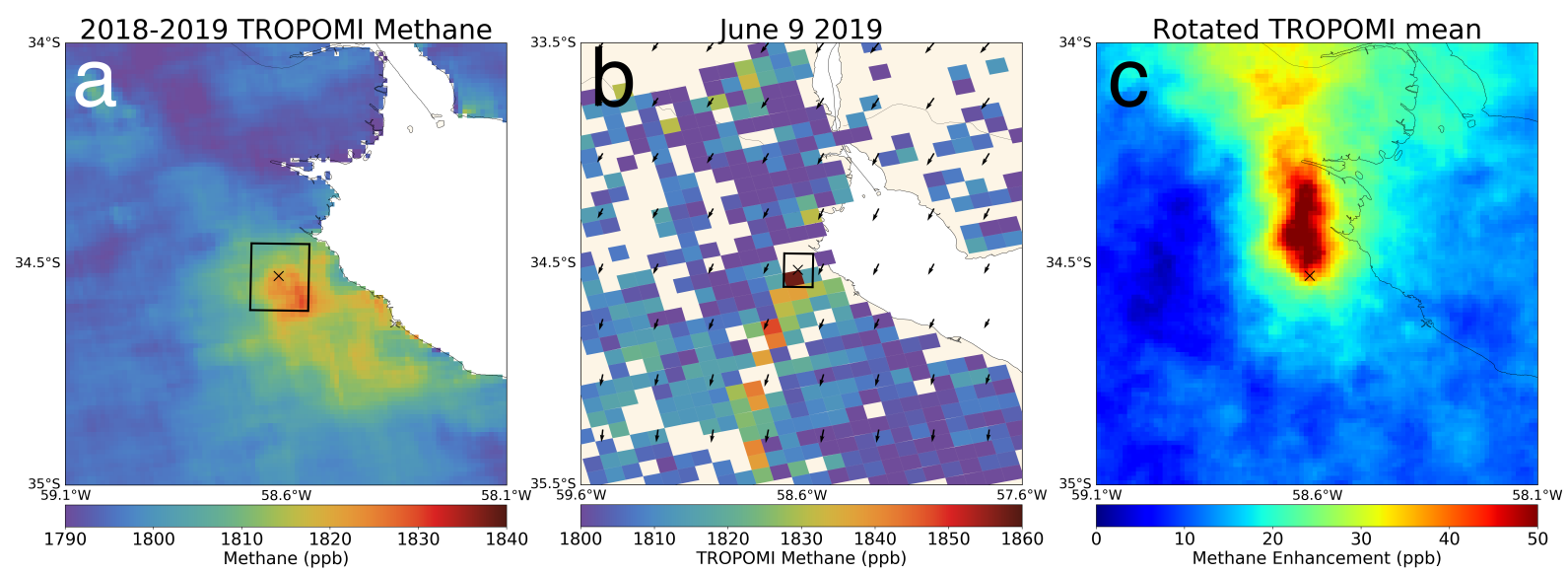

Figure 1: TROPOMI observations over Buenos Aires (Argentina) with (a) Mean 2018-2019 TROPOMI methane concentrations oversampled (i.e. accounting for the full footprint of the observation) on a $0.01^{\circ}$ grid. The Norte III landfill is indicated by the black cross; also shown is a GHGSat window centered on the TROPOMI-derived target. (b) A single TROPOMI overpass on June 92019 exhibiting a methane plume downwind of Buenos Aires with wind arrows representing ERA5 10-m winds (26). (c) The 2018-2019 wind-rotated average giving a clear (north-oriented) plume signal indicating a concentrated source.

lution of up to approximately $25 \times 25 \mathrm{~m}^{2}$ (25). When targeting locations with enhanced methane concentrations detected by TROPOMI, the GHGSat satellites can be used to identify individual sources and quantify their emissions. With its global coverage, TROPOMI can therefore guide ('tip and cue') target-mode observations and provide a powerful tool to identify strong point sources when combined with instruments like GHGSat. Because the GHGSat field of view is similar to the footprint of a single TROPOMI observation, TROPOMI data from multiple days need to be analyzed alongside wind information to determine the target locations with sufficient spatial precision.

We use long-term averages of TROPOMI methane data (22) to identify locations with persistently enhanced methane concentrations. Some of these locations have been shown to align with areas of known oil/gas production $(10,27)$ or coal mining $(28)$, but we also frequently find large enhancements over urban areas, such as Buenos Aires (Argentina; Figure 1a). To 
identify the best target point for GHGSat within these (often spread-out) hot spots, we use a wind-rotation technique. For a potential target point, we rotate the data on individual days (e.g., Figure $1 \mathrm{~b}$ ) based on the wind direction at $10 \mathrm{~m}$ from the ERA5 reanalysis meteorological fields (26), such that the wind vector is always oriented to the north. Where the target aligns with the methane source, the downwind concentrations are consistently enhanced compared to those upwind, resulting in a northward-oriented plume signal in the oversampled average of the rotated data (Figure 1c) (29-31). By evaluating wind-rotations for a dense grid of rotation points covering the area of interest, we can find which rotated average shows the largest downwind enhancement and thus pinpoint the source's location to within a few kilometers (Supplement 1). We apply this wind-rotation method to 2018-2019 TROPOMI data over Buenos Aires and find the optimal target $\left(34.53^{\circ} \mathrm{S}, 58.60^{\circ} \mathrm{W}\right)$ within $2 \mathrm{~km}$ of the Norte III landfill. We follow the same procedure for Delhi (India), Lahore (Pakistan), and Mumbai (India), where we also identify landfills as optimal targets for GHGSat observations (Supplement 1).

Figure 2 shows a sample of typical methane plumes detected by GHGSat-C1/C2 from the Norte III (Buenos Aires), Lakhodair (Lahore), Kanjurmarg (Mumbai), and Ghazipur (Delhi) landfills. Plume shapes are generally consistent with the wind direction from the GEOS-FP meteorological reanalysis. Using GEOS-FP wind speeds in an integrated mass enhancement (IME) calculation $(33,34)$ recalibrated for area sources (Supplement 2) we estimate mean methane emission rates between 3 and $29 \mathrm{t} \mathrm{hr}^{-1}$ for the four landfills (Table 1; full time series including GHGSat-D observations starting in December 2019 are shown in Figure S.7). Whenever there are clear-sky GHGSat-C1/C2 observations over the four sites, we detect emission plumes. Uncertainty in the estimated emissions (Supplement 2) and uncertainty in wind direction increase with decreasing wind speed (35), which can be seen from the mismatch between plume direction and wind vector for the 16 February 2020 observation of the Ghazipur landfill (Figure 2d). 

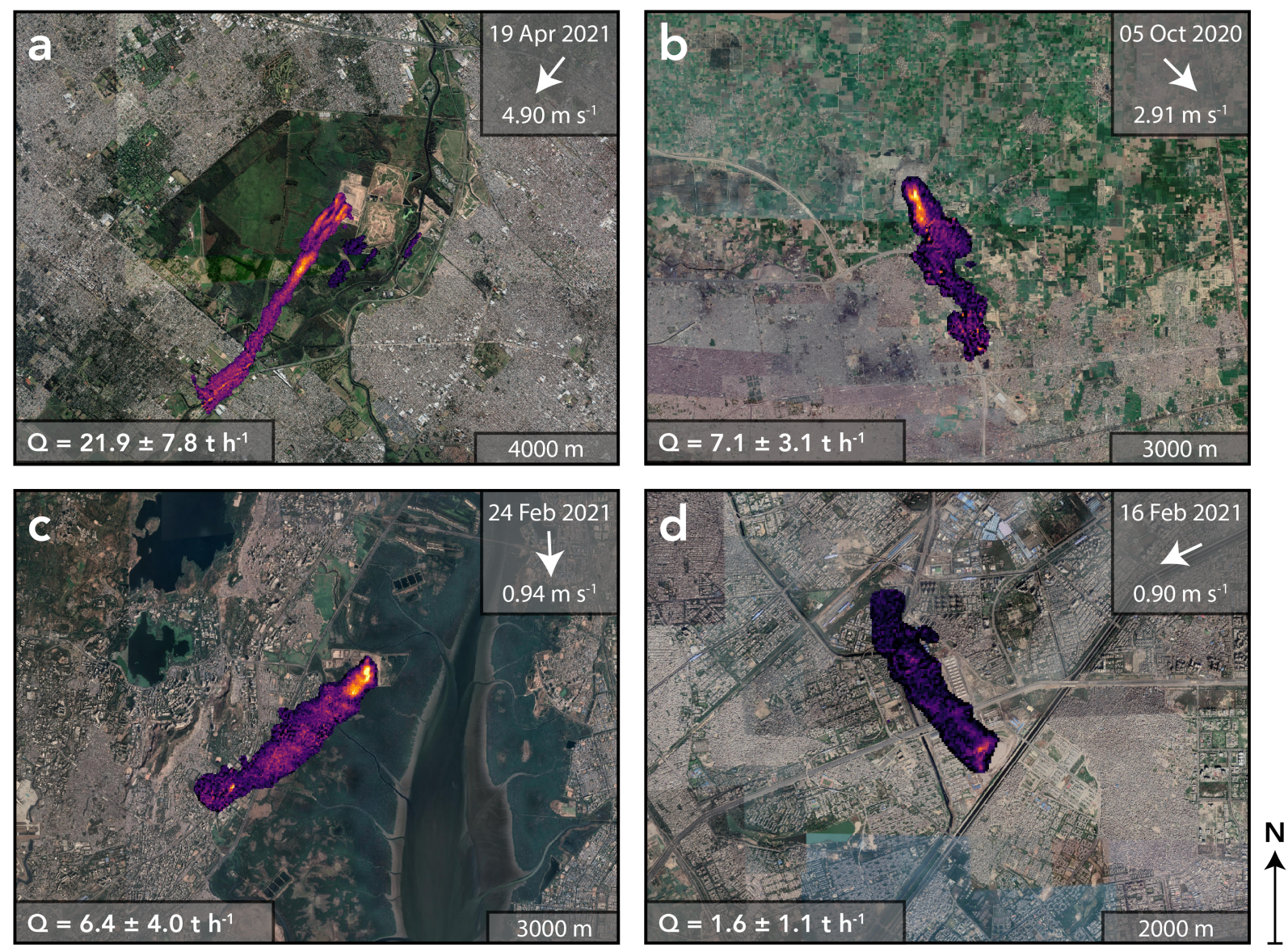

Background imagery @2020 Google, CNES/Airbus, Maxar Technologies

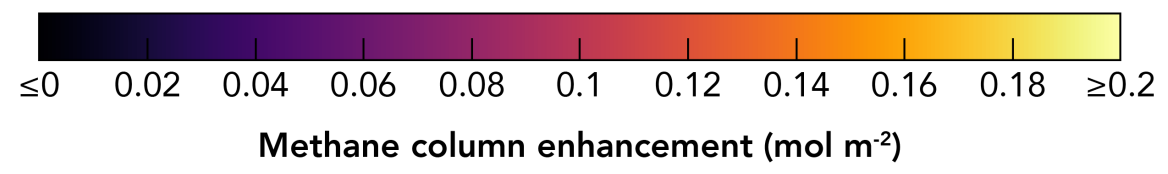

Figure 2: Methane plumes observed by GHGSat-C1/C2 from the (a) Norte III (Buenos Aires, Argentina), (b) Lakhodair (Lahore, Pakistan), (c) Kanjurmarg (Mumbai, India), and (d) Ghazipur (Delhi, India) landfills, in 2020 and 2021. Concentrations are plotted over aerial imagery. Wind directions are from GEOS-FP (32) and emission quantifications (Supplement 2) are shown in the legend. The leftmost plume in panel (a) is truncated at the edge of the viewing domain and quantified at $19.1 \pm 6.7 \mathrm{t} \mathrm{hr}^{-1}$; the other plume from the landfill and plume across the river are quantified at $2.7 \pm 1.0 \mathrm{t} \mathrm{hr}^{-1}$ and $1.6 \pm 0.6 \mathrm{t} \mathrm{hr}^{-1}$, respectively. The plume across the river is not incorporated in the estimate of the landfill's total emissions. 
The mean emissions we find for the Ghazipur landfill are within the 1.4-3.3 $\mathrm{t} \mathrm{hr}^{-1}$ range found for 2015 using emission models (36).

The fine resolution of GHGSat observations permits attribution of emissions to different sections of the landfills. While emissions from the Indian and Pakistani landfills appear widely distributed across the sites, emissions from the Norte III landfill originate mainly from the active module on the western side. This part of the landfill accounts for $87 \%$ of the detected emissions shown in Figure 2 and only has intermediate covering whereas the older eastern areas of the landfill were covered and closed in 2014 and 2018 using a $1.2 \mathrm{~m}$ cover with an active gas collection system. Emissions originate specifically from the two active surfaces on the northwest and southwest of the active module (Supplement Figure S.11) that receive waste from the city and province of Buenos Aires, respectively. At the active surfaces, waste is continuously deposited and the intermediate cover is relocated due to waste being added. Several vent wells have been installed as temporary mitigation tools in the active module but the active surfaces provide the largest windows for landfill gas to escape. GHGSat-D observations from February 2020 show isolated plumes from the individual surfaces on different days (Figure S.8). Because of the complicated nature of methane migration through the landfill, emissions are difficult to predict and no measurements are taken on the ground to characterize them. The GHGSat imagery shown here demonstrates how satellites can add information at a spatial scale finer than that of inventory calculations.

Total methane generation reported by the Norte III landfill for 2019 is equivalent to $16.5 \mathrm{t}$ $\mathrm{h}^{-1}$ and is calculated based on the UNFCCC methodology (37) incorporating landfill-specific information on the disposed waste, landfill architecture, methane fraction, and climate. Emissions are calculated per module of the landfill and the methane generation estimate does not 
take into account the gas extraction for the closed modules, which should significantly decrease net emissions. Whereas total methane generation is close to the GHGSat emission estimates (which average $29 \mathrm{t} \mathrm{hr}^{-1}$ but $21 \mathrm{t} \mathrm{hr}^{-1}$ excluding the highest quantification as an outlier), emissions from the active module (which has an extraction system under construction) are reported at just $4.3 \mathrm{t} \mathrm{h}^{-1}$ for 2019. The GHGSat observations (2020-2021) therefore indicate that emissions from the active surface may be underestimated while emissions from the closed modules are much lower and not always detected as plumes. On the two days where individual closedmodule plumes are detected, they account for only 8-13\% of total emissions from the landfill. This shows the covering and extraction system are largely successful and that emissions from the landfill could decrease quickly once the active surfaces are closed.

Table 1: City-level and facility-level emissions for the four landfills quantified using TROPOMI and GHGSat observations respectively $\left(\mathrm{t} \mathrm{hr}^{-1}\right)$.

\begin{tabular}{l|llll} 
& Buenos Aires & Delhi & Lahore & Mumbai \\
\hline City-level inventory $^{a}$ & 22 & 28 & 25 & 17 \\
City-level TROPOMI $^{b}$ & $61(57-67)$ & $50(42-55)$ & $58(47-63)$ & $28(15-30)$ \\
Facility-level GHGSat $^{c}$ & $28.6(15.8-57.8)$ & $2.6(1.6-3.8)$ & $6.4(2.3-16.0)$ & $9.8(6.1-26.0)$ \\
Landfill contribution & $47 \%$ & $5 \%$ & $11 \%$ & $35 \%$ \\
\hline
\end{tabular}

${ }^{a}$ Inventory (bottom-up) estimates are the sum of 2016 oil/gas/coal emissions from Scarpelli et al. (38), other 2015 anthropogenic emissions from EDGAR v5 (39), and 2017 wetland emissions from WetCHARTS v1.2.1 (40). Cities are taken as a $0.8^{\circ}$ box centered on the population-weighted centroid of the city.

${ }^{b}$ TROPOMI-based estimates are the result of an inversion using 2020 data. Ranges give the range of the inversion ensemble (Supplement 3).

${ }^{c}$ GHGSat estimates are based on the average of IME quantifications using GHGSat-C1/C2 data from 2019-2021. Ranges represent the spread of the individual quantifications. The Buenos Aires estimate includes estimates from the active and closed modules.

To put these emissions in context, we also quantify emissions from the surrounding urban areas using 2020 TROPOMI data. Emissions are estimated using the Weather Research and Forecasting chemical transport model (WRF-Chem (41)) at 3-km resolution, scaling inventory 
emissions (38-40) in a gridded Bayesian inversion to obtain the best match between simulated concentrations and TROPOMI observations (Supplement 3). The resulting urban emissions are given in Table 1. We find that commonly used emission inventories underestimate Buenos Aires's 2020 urban emissions by a factor 2.8 and those of the other cities by factors 1.6-2.3. Based on the mean of the GHGSat observations, the observed landfills are responsible for 5\%$47 \%$ of the city-wide emissions. In Buenos Aires and Mumbai, the individual landfills account for more than a third of total urban emissions. The Norte III landfill makes up about half of Argentina's solid waste emissions ( $49 \mathrm{t} \mathrm{hr}^{-1}$, with $26 \mathrm{t} \mathrm{hr}^{-1}$ coming from managed landfills) reported to the UNFCCC for 2016 (42), which is not unexpected as the Buenos Aires province houses $40 \%$ of Argentina's population (43). The Lakhodair landfill alone accounts for $10 \%$ of the 2015 UNFCCC-reported solid waste emissions for Pakistan (44), despite the Lahore district making up only $5 \%$ of the country's population (45). This reflects a need to refine the magnitude and spatial representation of landfill and urban emissions in global inventory databases.

The complementarity of TROPOMI and GHGSat provides a powerful tool to detect, locate, and quantify emissions from strong methane point sources around the world. Detections can be used to inform operators and regulators, and promote action on cost-effective methane emission reduction measures. After identification of the emitting facility, continued observation allows monitoring of emissions and evaluation of mitigation measures. The hybrid methodology demonstrated here can also be applied with the successors of TROPOMI (e.g. Sentinel-5) and be used to guide current and future target-mode hyper-spectral instruments (e.g. the Precursor and Application Mission (PRISMA) $(12,46)$ and the Environmental Mapping and Analysis Program (EnMAP) (47)) or inspection of imagery from global-surveying high-resolution multispectral instruments (e.g. Sentinel-2, Landsat $(27,48)$ ). Combining these diverse data streams enables global identification of strong methane sources followed by facility-level monitoring 
necessary to reduce emissions in the short term, improve emissions inventories for climate policy, and support regulatory enforcement.

\section{References}

1. D. Shindell, et al., Science 335, 183 (2012).

2. A. Stohl, et al., Atmospheric Chemistry and Physics 15, 10529 (2015).

3. E. G. Nisbet, et al., Reviews of Geophysics 58, e2019RG000675 (2020). E2019RG000675 2019RG000675.

4. United Nations Environmental Programme, Global methane assessment: Benefits and costs of mitigating methane emissions, Available at: https://www.unep.org/resources/report/ global-methane-assessment-benefits-and-costs-mitigating-methane-emissio (2021).

5. NOAA/GML, NOAA Global Monitoring Laboratory: Trends in Atmospheric Methane, Available at: https://www.esrl.noaa.gov/gmd/ccgg/trends_ch4/, last access 4/10/2021 (2021).

6. D. Zavala-Araiza, et al., Nature communications 8, 1 (2017).

7. R. M. Duren, et al., Nature 575, 180 (2019).

8. D. J. Jacob, et al., Atmospheric Chemistry and Physics 16, 14371 (2016).

9. R. A. Alvarez, et al., Science 361, 186 (2018). 
10. D. J. Varon, et al., Geophysical Research Letters 46, 13507 (2019).

11. S. Pandey, et al., Proceedings of the National Academy of Sciences 116, 26376 (2019).

12. D. H. Cusworth, et al., Geophysical Research Letters 48, e2020GL090864 (2021). E2020GL090864 2020GL090864.

13. J. A. de Gouw, et al., Scientific reports 10, 1 (2020).

14. Y. Zhang, et al., Science Advances 6 (2020).

15. O. Schneising, et al., Atmospheric Chemistry and Physics 20, 9169 (2020).

16. M. Saunois, et al., Earth System Science Data 12, 1561 (2020).

17. UNFCCC, United Nations Framework Convention on Climate Change: Greenhouse Gas Inventory Data, Described at: https://unfccc.int/ process/transparency-and-reporting/greenhouse-gas-data/ ghg-data-unfccc, obtained from http://di.unfccc.int/detailed_ data_by_party (2019).

18. S. Kaza, L. Yao, P. Bhada-Tata, F. Van Woerden, What a waste 2.0: a global snapshot of solid waste management to 2050 (World Bank Publications, 2018).

19. L. Höglund-Isaksson, A. Gómez-Sanabria, Z. Klimont, P. Rafaj, W. Schöpp, Environmental Research Communications 2, 025004 (2020).

20. T. B. Thomasen, C. Scheutz, P. Kjeldsen, Waste Management 84, 29 (2019).

21. J. Veefkind, et al., Remote Sensing of Environment 120, 70 (2012). The Sentinel Missions - New Opportunities for Science. 
22. A. Lorente, et al., Atmospheric Measurement Techniques 14, 665 (2021).

23. D. Jervis, et al., Atmospheric Measurement Techniques Discussions pp. 1-23 (2020).

24. A. Ramier, et al., AGU Fall Meeting Abstracts (2020), vol. 2020, pp. A247-03.

25. M. Ligori, L. Bradbury, R. Spina, R. E. Zee, S. Germain, GHGSat constellation: The future of monitoring greenhouse gas emissions (2019).

26. H. Hersbach, et al., Quarterly Journal of the Royal Meteorological Society 146, 1999 (2020).

27. I. Irakulis-Loitxate, L. Guanter, J. D. Maasakkers, D. Zavala-Araiza, I. Aben, EarthArXiv (2021).

28. P. Sadavarte, et al., Environmental Science \& Technology 55, 16573 (2021). PMID: 34842427.

29. M. Pommier, C. A. McLinden, M. Deeter, Geophysical Research Letters 40, 3766 (2013).

30. L. Zhu, et al., Environmental Science \& Technology 51, 5650 (2017). PMID: 28441488.

31. L. Clarisse, M. V. Damme, C. Clerbaux, P.-F. Coheur, Atmospheric Measurement Techniques 12, 5457 (2019).

32. A. Molod, et al., The geos-5 atmospheric general circulation model: Mean climate and development from merra to fortuna (2012).

33. C. Frankenberg, et al., Proceedings of the national academy of sciences 113, 9734 (2016).

34. D. J. Varon, et al., Atmospheric Measurement Techniques 11, 5673 (2018). 
35. D. J. Varon, D. J. Jacob, D. Jervis, J. McKeever, Environmental Science \& Technology 54, 10246 (2020). PMID: 32672947.

36. P. Ghosh, et al., Bioresource technology 272, 611 (2019).

37. H. Eggleston, L. Buendia, K. Miwa, T. Ngara, K. Tanabe, 2006 ipcc guidelines for national greenhouse gas inventories (2006).

38. T. R. Scarpelli, et al., Earth System Science Data 12, 563 (2020).

39. M. Crippa, et al., Scientific data 7, 1 (2020).

40. A. A. Bloom, et al., Geoscientific Model Development 10, 2141 (2017).

41. J. G. Powers, et al., Bulletin of the American Meteorological Society 98, 1717 (2017).

42. Secretariía de Ambiente y Desarrollo Sustentable República Argentina, Tercer informe bienal de actualización de la república argentina a la convención marco de las naciones unidas sobre el cambio climático, Available at: https://unfccc.int/documents / 201965 (2019).

43. I. N. de Estad'istica y Censos, Censo nacional de poblaci'on, hogares y viviendas 2010, Available at: https://www.indec.gob.ar/ftp/cuadros/poblacion/ censo2010_tomo2.pdf, last access 4/10/2021 (2012).

44. Government of Pakistan Ministry of Climate Change, Pakistan's second national communication on climate change, Available at: https://unfccc.int/documents/ 199292 (2019).

45. P. B. of Statistics, Block wise provisional summary results of 6th population \& housing census-2017, Available at: https://www.pbs.gov.pk/content/ 
block-wise-provisional-summary-results-6th-population-housing-census-20 last access 4/17/2021 (2018).

46. R. Loizzo, et al., Prisma: The italian hyperspectral mission, igarss 2018-2018 ieee international geoscience and remote sensing symposium, 175-178 (2018).

47. L. Guanter, et al., Remote Sensing 7, 8830 (2015).

48. D. J. Varon, et al., Atmospheric Measurement Techniques 14, 2771 (2021).

49. D. Wunch, et al., Philosophical Transactions of the Royal Society of London A: Mathematical, Physical and Engineering Sciences 369, 2087 (2011).

50. S. Jongaramrungruang, et al., Atmospheric Measurement Techniques 12, 6667 (2019).

51. N. C. for Environmental Prediction, Ncep fnl operational model global tropospheric analyses, https://doi.org/10.5065/D6M043C6 (2000).

52. E. N. Koffi, P. Bergamaschi, Evaluation of copernicus atmosphere monitoring service methane products, Tech. Rep. EUR 29349 EN, European Commission Joint Research Centre (2018).

53. T. Gumbricht, et al., Tropical and Subtropical Wetlands Distribution version 2 (2017).

54. J. D. Maasakkers, et al., Atmospheric Chemistry and Physics 19, 7859 (2019).

\section{Acknowledgements}

The authors thank the team that realized the TROPOMI instrument and its data products, consisting of the partnership between Airbus Defence and Space Netherlands, KNMI, SRON, and 
TNO, commissioned by NSO and ESA. Sentinel-5 Precursor is part of the EU Copernicus program, Copernicus (modified) Sentinel-5P data (2018-2020) have been used. Part of this work was carried out on the Dutch national e-infrastructure with the support of SURF Cooperative. G.M. was funded by the ESA Earthnet Data Assessment Pilot (EDAP). A.L., T.B., P.T., and T.A.v.K. are funded by the TROPOMI national program from the NSO. S.P. is funded through the GALES project (\#15597) by the Dutch Technology Foundation STW, which is part of the Netherlands Organization for Scientific Research (NWO), and is partly funded by the Ministry of Economic Affairs. A.E. was funded by the Harvard University Alex G. Booth Fellowship. We thank Rodrigo Martín Pontiggia and Jorgelina Laura Blanco from the Innovation team at Benito Roggio Ambiental S.A. for providing information on the Buenos Aires landfill.

\section{Data availability}

TROPOMI data are available at https://ftp.sron.nl/open-access-data-2/ TROPOMI/tropomi/ch4/14_14_Lorente_et_al_2020_AMTD/. Example GHGSat-D/C1/C2 methane column data are available upon request. The WRF-Chem model code can be downloaded at https://ruc.noaa.gov/wrf/wrf-chem/. GEOSFP wind data can be downloaded at gmao.gsfc.nasa.gov/GMAO_products. ERA5 wind data are available at https://cds.climate.copernicus.eu. The NCEP meteorology are available at https://rda.ucar.edu/datasets/ds094.0/. The CAMS boundary conditions are available at https://apps.ecmwf.int/datasets/data/ cams-nrealtime/levtype=pl/. EDGAR v5.0 emissions are available at edgar. jrc.ec.europa.eu/overview.php?v=50_GHG, Scarpelli et al. (38) emissions can be retrieved from https://doi.org/10.7910/DVN/HH4EUM, and WetCHARTs emissions are available at https://daac.ornl.gov/cgi-bin/dsviewer.pl?ds_id=1502. 
Additional information related to this paper may be requested from the authors. 


\section{Supplementary materials}

\section{Supplement 1: TROPOMI data and source localization}

The Tropospheric Monitoring Instrument (TROPOMI) is a pushbroom spectrometer that was launched aboard the Sentinel-5P satellite in October 2017 (21,22). It retrieves methane with daily global coverage from the $2305-2385 \mathrm{~nm}$ shortwave infrared (SWIR) band and the (757$774 \mathrm{~nm}$ ) near-infrared (NIR) band with $5.5 \times 7 \mathrm{~km}^{2}$ resolution at nadir and a swath width of $\sim 2600 \mathrm{~km}$ at an overpass time of around 13:30 local time.

We use the TROPOMI methane product described in Lorente et al. (2021) that shows good agreement (-3.4 ppb average bias with $5.6 \mathrm{ppb}$ station-to-station variability) with the Total Carbon Column Observing Network (TCCON) (49). For the source localization and the TROPOMI-based emission quantification (Supplement 3), we use albedo-bias corrected data over land with: methane precision $<10 \mathrm{ppb}$; SWIR cloud fraction $<0.02 ;$ SWIR aerosol optical depth $<0.1 ;$ SWIR albedo $>0.02$.

To identify regions of interest we oversample $2018-2019$ TROPOMI data at $0.01^{\circ} \times 0.01^{\circ}$ resolution following Zhu et al. (2017) where the full spatial footprint of the observation is taken into account by attributing the observed value to grid cells weighted by the spatial overlap of the observation with those grid cells. For a region to be of interest, we filter based on the enhancement (defined as the difference between the TROPOMI retrieval and the a priori used in the retrieval); require sufficient coverage at the considered grid cell as well as surrounding grid cells to filter anomalous values at the edges of the TROPOMI coverage; and require limited local correlation with SWIR albedo, SWIR aerosol optical depth, and coverage (Figure S.1). Multiple regions of interest result, related to various emission sources but here we focus on the 

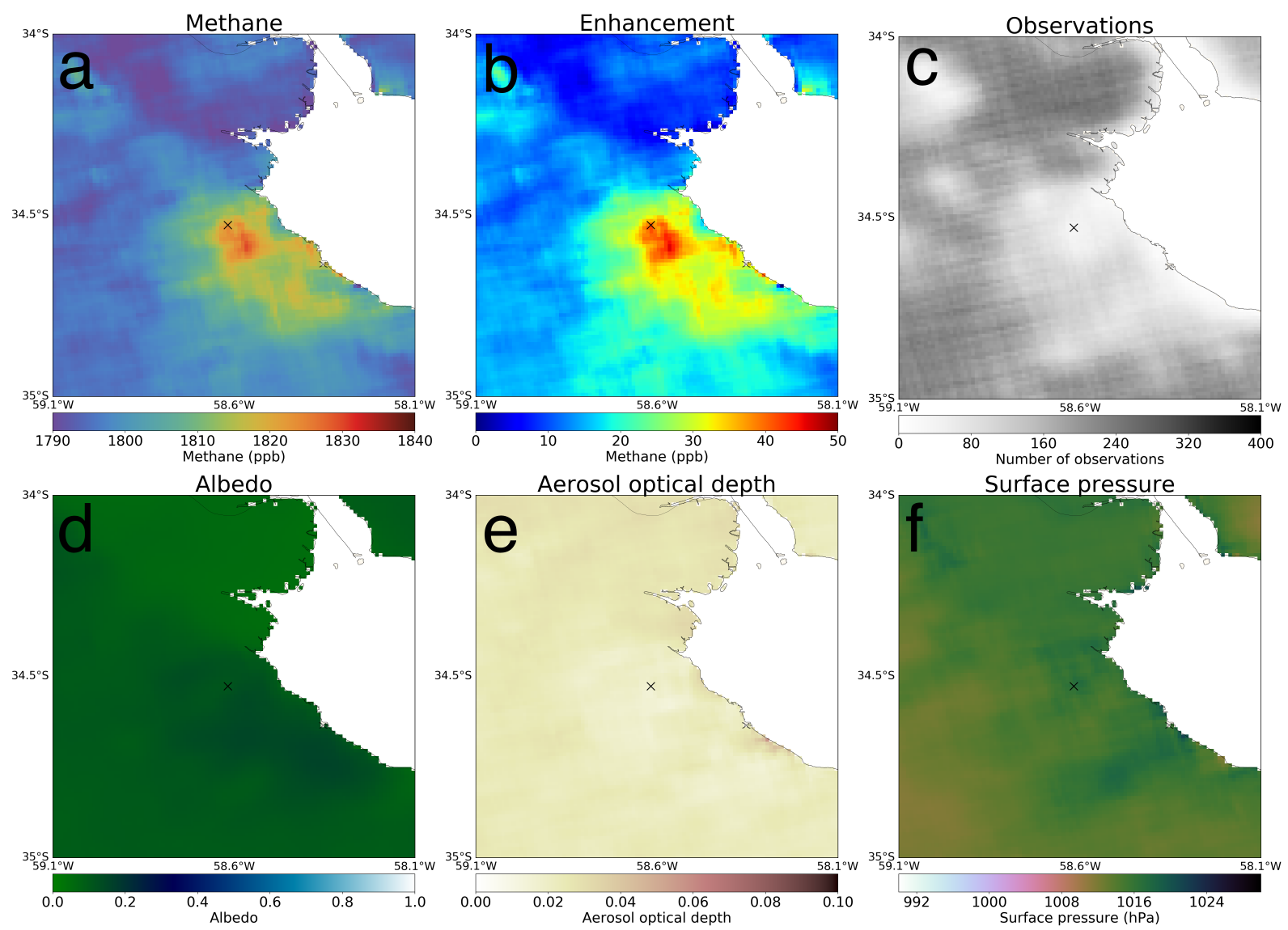

Figure S.1: Oversampled 2018-2019 TROPOMI methane data over Buenos Aires (a) as well as supporting data used in the source localization procedure: methane enhancement (b), number of observations (c), SWIR surface albedo (d), SWIR aerosol optical thickness (e), and surface pressure (f).

Buenos Aires (Argentina), Delhi (India), Lahore (Pakistan), and Mumbai (India) urban areas.

After identifying a location of interest, we use a wind-rotation technique $(29,31)$ to pinpoint the potential target location with sufficient precision so the source will fall within GHGSat's $\sim$ $10 \times 10 \mathrm{~km}^{2}$ field-of-view when targeting this location. The rationale behind this method is that simply averaging the TROPOMI data will result in smearing of signal due to varying wind directions on different days. Rotating (TROPOMI) methane enhancements around a source location (such that the wind is always pointing north) will lead to aligning plumes on differ- 
ent days. This feature is the result of downwind concentrations always being larger than upwind concentrations at a source location. The rotated 2018-2019 data are then oversampled at $0.01^{\circ}$ resolution, resulting in an average downwind "plume-like" signal. We perform this windrotation for a full grid of $13 \times 13$ points, first distanced at $0.05^{\circ}$ (Shown for Buenos Aires in Figure S.2) and subsequently at $0.01^{\circ}$ (Figure S.3) to determine which location has the largest downwind enhancement and hence is the most likely location of the source. Wind data come from a spline interpolation of the hourly $10-\mathrm{m}$ wind field closest in time to the TROPOMI overpass from the $0.25^{\circ} \times 0.25^{\circ}$ ERA5 reanalysis product $(26)$.

To analyze which rotated image is centered at the most likely source location, we compute several metrics based on the oversampled averages (Figure S.4). These metrics are: the mean enhancement in a $25 \times 5 \mathrm{~km}^{2}$ box downwind of the source; the difference between that enhancement and the enhancement in the $25 \times 5 \mathrm{~km}^{2}$ box upwind; and the maximum concentration downwind of the source. We look at the agreement between these metrics to estimate the most likely source location, usually best represented by the mean downwind concentration. For Buenos Aires, the optimized location is $34.53^{\circ} \mathrm{S}, 58.60^{\circ} \mathrm{W} \pm 0.01^{\circ}$, all optimized locations are given in Table S.1. The reported uncertainty of estimated location is based on the absolute distance between the two most separated points where the metrics peak. Where necessary, locations can be fine-tuned using external information from visual imagery or emission databases to ensure the most likely (or maximum number of) emission targets are within the GHGSat field of view. This is for example necessary in Lahore where there is a large diffuse background emission from the city. Mean TROPOMI concentrations and rotated averages centered at the landfills for the three other targets are shown in Figure S.5. 
Wind-rotated data grid over Buenos Aires

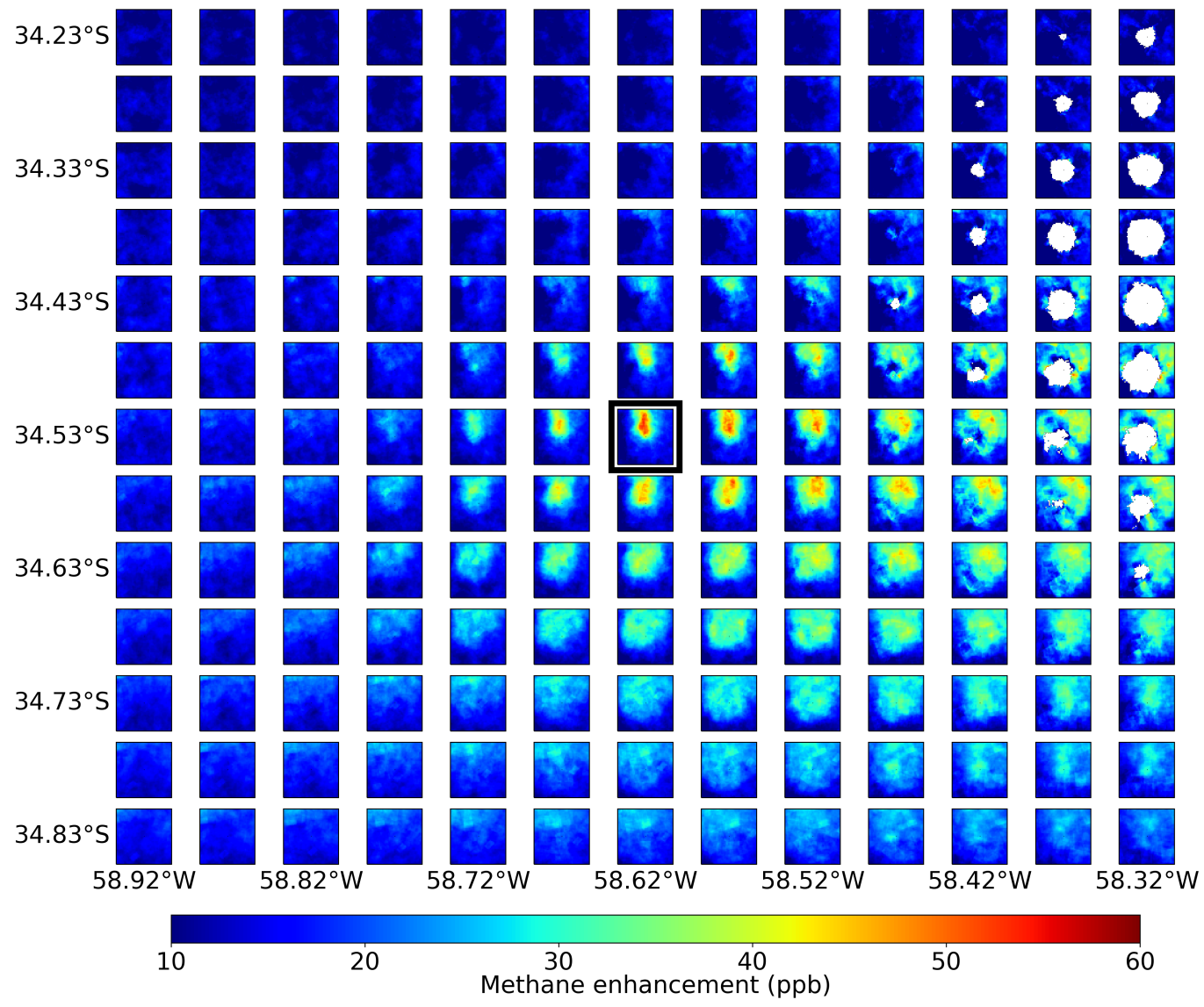

Figure S.2: Rotated and oversampled 2018-2019 TROPOMI methane enhancements for a grid of rotation points separated by $0.05^{\circ}$ spanning a region of $0.6^{\circ} \times 0.6^{\circ}$ centered at the Norte III landfill in Buenos Aires. The image centered on the landfill is indicated by a black box. Each individual rotated image shows an area of $1^{\circ} \times 1^{\circ}$. Areas with fewer than ten TROPOMI observations are shown in white. 
Wind-rotated data grid over Buenos Aires

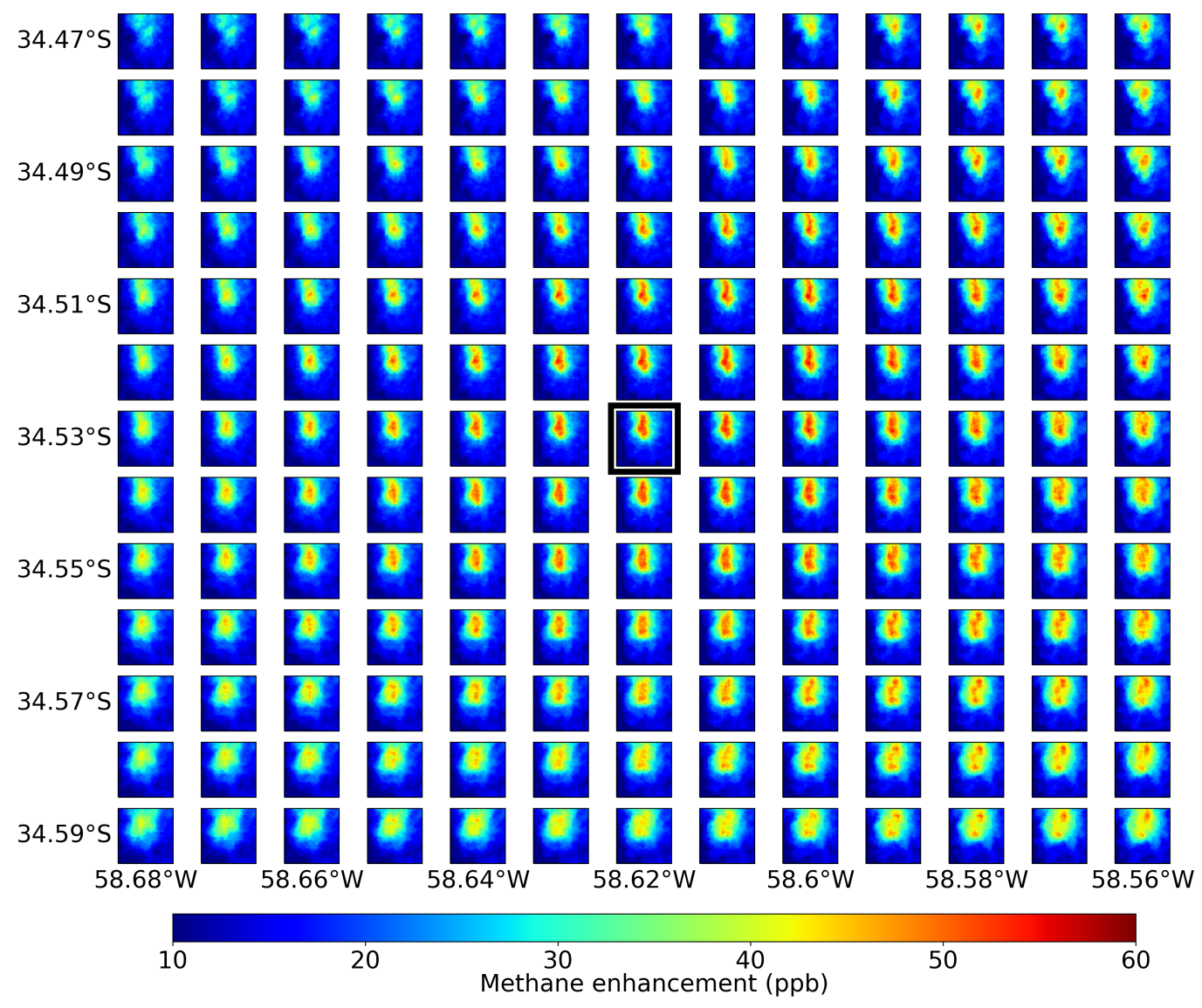

Figure S.3: Rotated and oversampled 2018-2019 TROPOMI methane enhancements for a grid of rotation points separated by $0.01^{\circ}$ grid covering an area of $0.12^{\circ} \times 0.12^{\circ}$ centered at the Norte III landfill in Buenos Aires. The image centered on the landfill is indicated by a black box. 

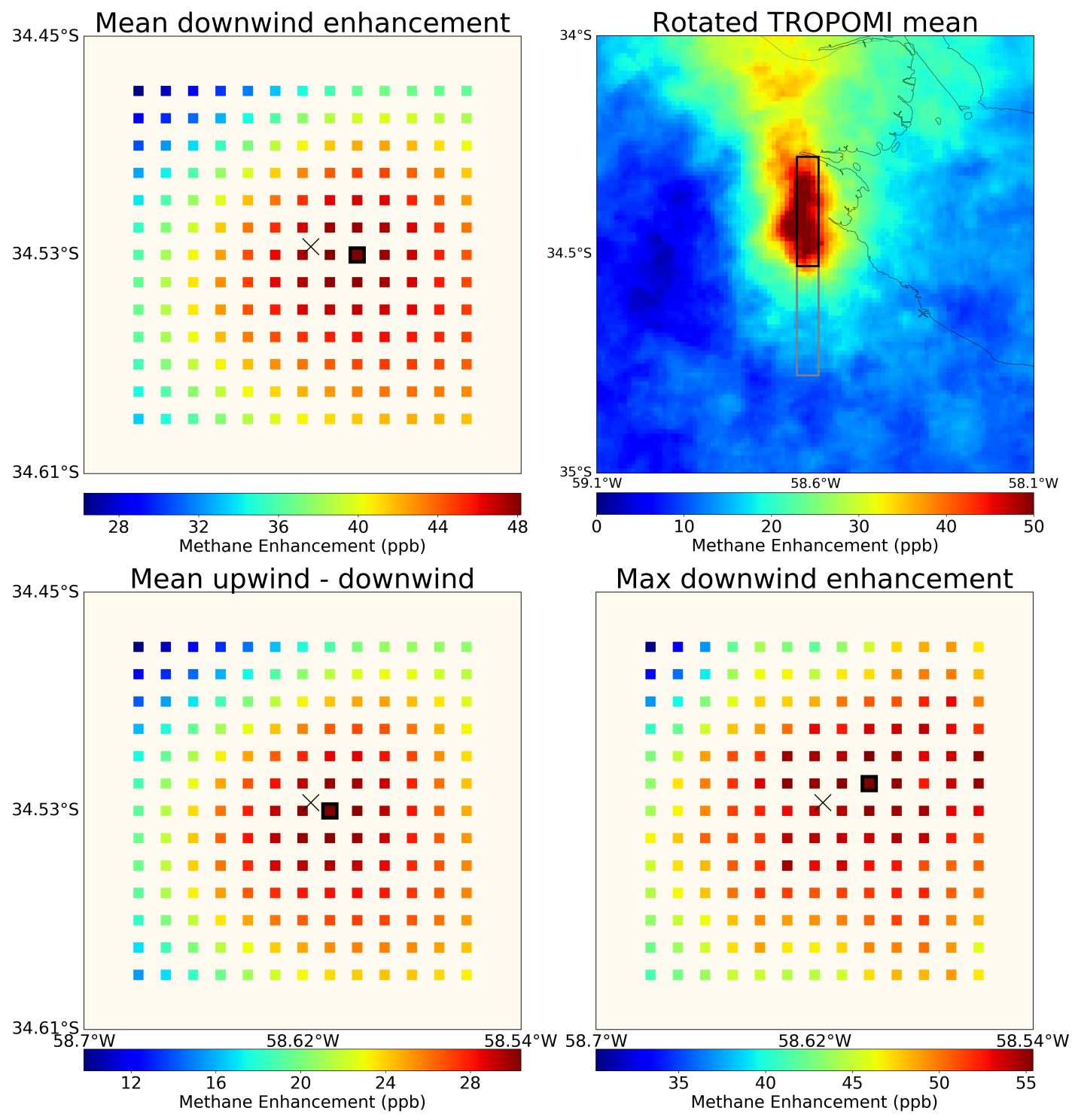

Figure S.4: Metrics used to evaluate the grid of wind rotations. The top right figure shows the $25 \times 5 \mathrm{~km}^{2}$ downwind (black) and upwind (grey) boxes used in the computations on top of the rotated plume centered at the Norte III landfill. The computed values are the mean enhancements in the downwind boxes (top left); the difference between those enhancements and their upwind equivalents (bottom left); and the maximum enhancements downwind of the source (bottom right). The location of the landfill in the metric panels is marked by the black cross, the locations with the highest metric values are indicated with a black outline. 

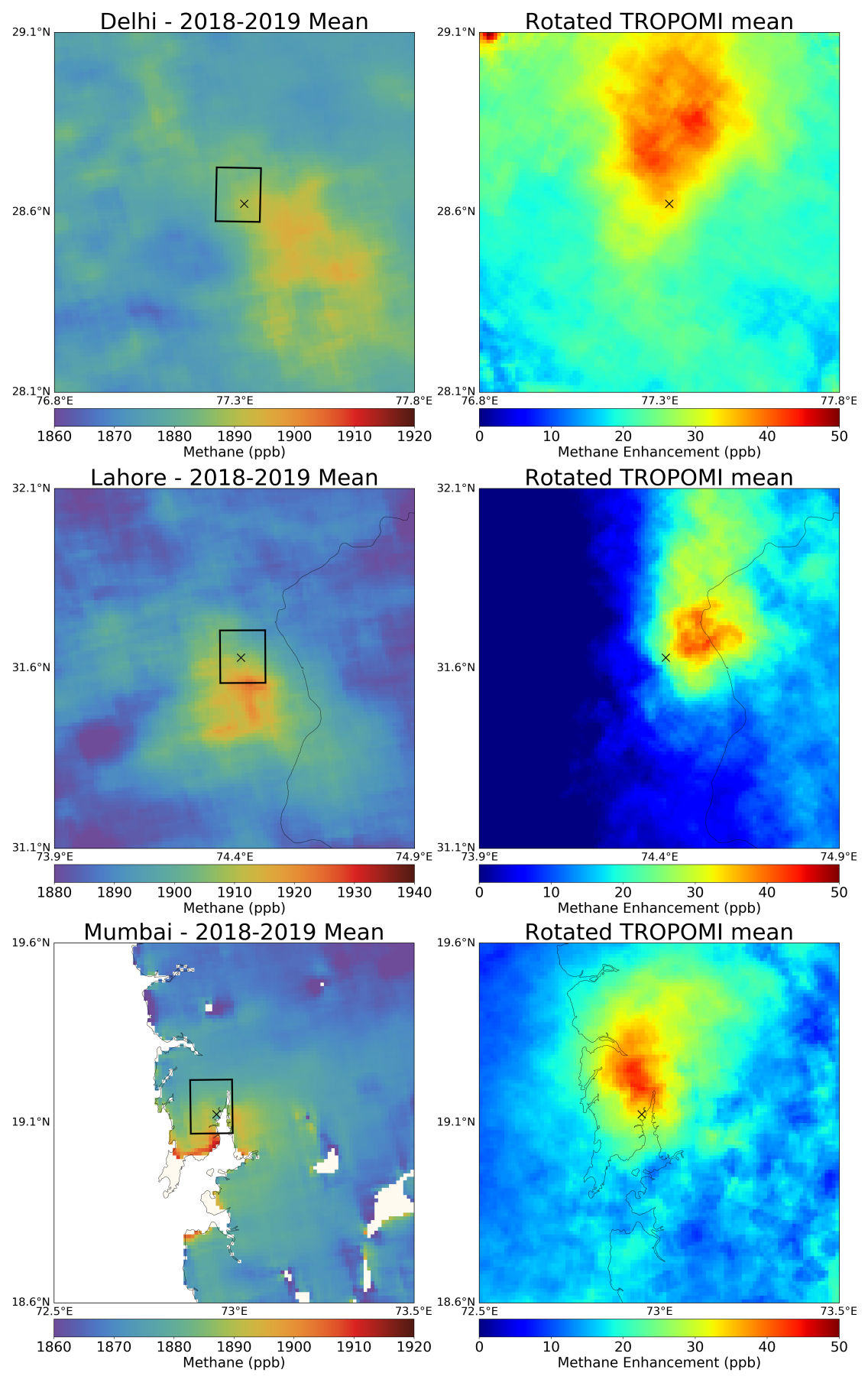

Figure S.5: TROPOMI observations over Delhi (India), Lahore (Pakistan), and Mumbai (India). The left column shows mean 2018-2019 TROPOMI methane concentrations oversampled on a $0.1^{\circ}$ grid. The targeted landfills are indicated by the black crosses, also shown are the targeted GHGSat windows. The right column shows 2018-2019 wind-rotated averages centered on the landfills. 
Table S.1: Optimized target locations using the wind-rotation method on TROPOMI data

\begin{tabular}{l|ll} 
& Optimized Location & Landfill Location \\
\hline Buenos Aires & $34.53^{\circ} \mathrm{S}, 58.60^{\circ} \mathrm{W} \pm 0.01^{\circ}$ & $34.53^{\circ} \mathrm{S}, 58.62^{\circ} \mathrm{W}$ \\
Delhi & $28.63^{\circ} \mathrm{N}, 77.37^{\circ} \mathrm{E} \pm 0.07^{\circ}$ & $28.62^{\circ} \mathrm{N}, 77.33^{\circ} \mathrm{E}$ \\
Lahore & $31.54^{\circ} \mathrm{N}, 74.32^{\circ} \mathrm{E} \pm 0.05^{\circ}$ & $31.63^{\circ} \mathrm{N}, 74.42^{\circ} \mathrm{E}$ \\
Mumbai & $19.09^{\circ} \mathrm{N}, 72.89^{\circ} \mathrm{E} \pm 0.03^{\circ}$ & $19.12^{\circ} \mathrm{N}, 72.95^{\circ} \mathrm{E}$ \\
\hline
\end{tabular}




\section{Supplement 2: GHGSat data, emission quantification, and uncertainty}

GHGSat satellite instruments are wide-angle imaging Fabry-Perot spectrometers that retrieve atmospheric methane columns by solar backscatter in the 1630-1675 nm shortwave infrared spectral range. The demonstration instrument GHGSat-D was launched in June 2016 and observes at around 10:00 local time, with a return time of two weeks. It has a targeted field-of-view of $\sim 10 \times 10 \mathrm{~km}^{2}$ with an effective pixel resolution of $50 \times 50 \mathrm{~m}^{2}$ and is described in detail in Jervis et al. (2021). Follow-up instruments GHGSat-C1 and GHGSat-C2 were launched in 2020 and 2021 with an improved detection limit and effective pixel resolution of approximately $25 \times 25 \mathrm{~m}^{2}(24)$.

We use the Integrated Mass Enhancement (IME) method $(7,33,34)$ calibrated with Large Eddy Simulations (LESs) to quantify emissions with GHGSat observations. Varon et al. (2018) calibrated IME source-rate retrievals using LESs of methane plumes originating from point sources. Here we expand on that calibration using a uniform square area source to estimate source rates for the landfill plumes detected by GHGSat (Figure 2). The method relates the source rate $Q$ to the $I M E$ of the plume:

$$
Q=\frac{U_{e f f} I M E}{L}
$$

where $U_{\text {eff }}=f\left(U_{10}\right)$ is an effective wind speed that can be expressed as a function of the local $10-\mathrm{m}$ wind speed $U_{10}$, and $L$ is a plume length scale commonly defined as the square root of the area $(A)$ of the detectable plume: $L=\sqrt{A}$. The plume area is calculated from a binary plume mask that distinguishes plume pixels from background pixels. Here we define the mask in the same way as Varon et al. (2019), by applying a threshold to the retrieved columns and smoothing the resulting mask. We use 10-m wind speeds from GEOS-FP (32). 
Calibrating the IME source-rate retrieval involves characterizing the effective wind speed for a set of measurement conditions, either as a function of $U_{10}(34)$, or based on the shape of the observed plume (50). Here we use the former approach and compute source rates by mapping $U_{10}$ as reported in the GEOS-FP meteorological database to an effective wind speed. We perform five 3-hour-long simulations of a $275 \times 275 \mathrm{~m}^{2}$ area source mimicking a landfill, using a variety of meteorological conditions. Our model setup is identical to that of Varon et al. (2021), but with an area source rather than a point source. The first hour of each simulation is used to spin up turbulence, and data from the last two hours are used to determine the relationship between $U_{10}$ and $U_{\text {eff }}$ in the IME method. In parallel, we also use the point-source simulation ensemble from Varon et al. (2021).

Drawing snapshots from these two LES ensembles in 30 second intervals, we obtain 1200 samples each of area- and point- source plumes. We scale the snapshots to reflect random source rates in the range $2-30 \mathrm{t} \mathrm{h}^{-1}$. We integrate the snapshots vertically and add synthetic measurement noise drawn from a normal distribution with mean zero and standard deviation $5 \%$ of a $1875 \mathrm{ppb}$ methane background. This noise level (retrieval precision) is determined from the GHGSat-D, -C1, and -C2 retrieval fields for the four landfills, as the average standard deviation of non-plume methane enhancements across all the observed scenes. In this manner we obtain 2400 GHGSat pseudo-observations of point-source and area-source plumes. We then follow the methodology of Varon et al. (2018) to derive effective wind speed functions from the two synthetic plume datasets.

Figure S.6 shows the resulting effective wind speed functions for area sources and point sources. We find that first degree polynomials capture the dependence well $\left(0.78<\mathrm{R}^{2}<0.86\right)$ 


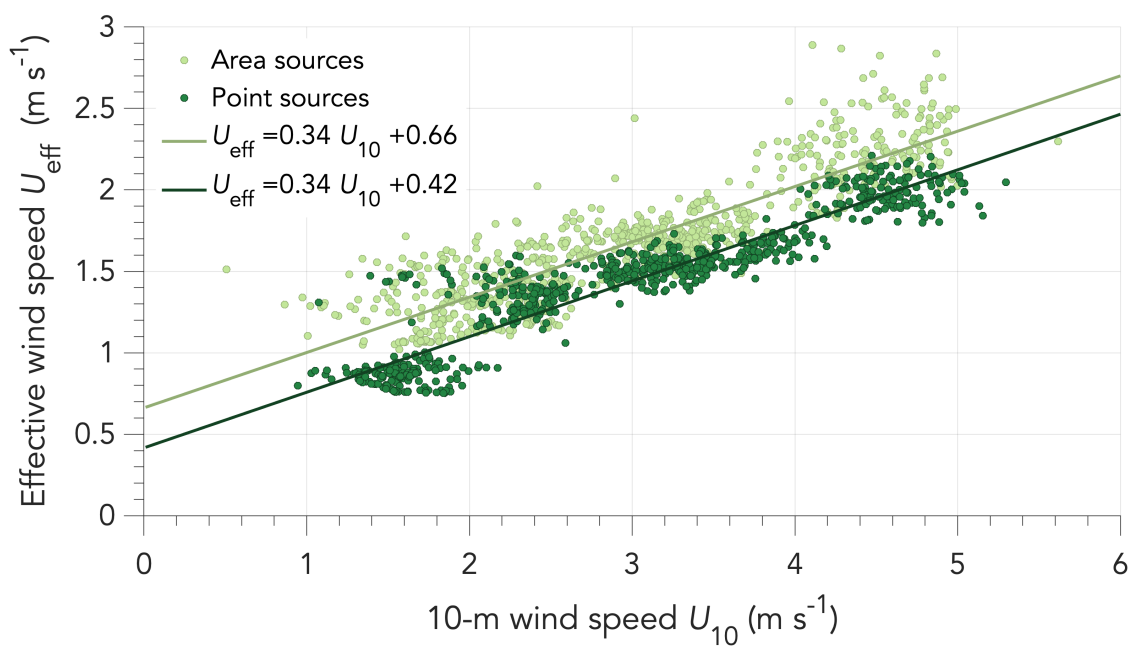

Figure S.6: Effective wind speed fits for LES area sources and point sources in the IME method, and associated linear regressions.

in both cases. The linear fits for the two populations are similar, but the effective wind speed is generally higher and more variable for area sources than for point sources. This is because area-source plumes are more diffuse and tend to have lower enhancements than point-source plumes of similar source strength. Weaker enhancements for area-source plumes are counterbalanced by higher effective wind speeds to recover the known $Q$ during calibration, and the reduced signal leads to higher uncertainty in the effective wind speed needed for each plume snapshot. Best-fit lines are computed by robust linear regression, which assigns less weight to outlier points, to mitigate the impact of marginally detectable LES plumes on the effective wind speed fit. For the same reason, Figure S.6 excludes plumes with IME below the 10th percentile of each LES ensemble.

Varon et al. (2018) found a similar range of effective and 10-m wind speeds for their LES methane point-source plumes, but a logarithmic dependence of $U_{\text {eff }}$ on $U_{10}$ rather than the 
linear dependence shown here. This may be due to differences in spatial resolution and/or meteorological settings between the Varon et al. (2018) LES ensemble and the ensembles used here. Source rate estimates using a linear or logarithmic fit are however similar for the range of wind speeds covered by the LES ensembles, where absolute differences are on average less than $6 \%$. Larger deviations can occur under low $\left(U_{10}<1.5 \mathrm{~m} \mathrm{~s}^{-1}\right)$ and high $\left(U_{10}>6 \mathrm{~m} \mathrm{~s}^{-1}\right)$ wind conditions. We use the area-source calibration of Figure S.6 to report best estimates for the landfill plumes observed by GHGSat (Figure 2), and the point-source calibration to estimate error from uncertainty in the source shape.

We estimate the uncertainty in our retrieved source rates similarly to Varon et al. (2019), accounting for wind speed error, model error in the IME method, and error from measurement noise. Here we include an additional error term for uncertainty in the shape and spatial extent of the source. The emissions detected by GHGSat may originate from a combination of gas extraction wells, active working faces, gaps in the landfill cover, and other potential methane sources at the target landfills. The true spatial distribution of the emissions may therefore be highly complex, but our source quantification scheme assumes emissions are distributed uniformly across a $275 \times 275 \mathrm{~m}^{2}$ area. To estimate the resulting error, for each LES plume we perform a separate source-rate retrieval calibrated with our point source ensemble, and compare the implied emission rate $Q_{p}$ with the result $Q_{a}$ from the area-source retrieval. We estimate the error from source shape uncertainty as the standard deviation of the differences between $Q_{a}$ and $Q_{p}$ for each landfill site, which comes to $<15 \%$ on average. Finally, we include an additional $10 \%$ error for using a single $U_{\text {eff }}$ function to retrieve source rates for a variety of different scenes and satellite instruments. This is a conservative estimate from a comparison of $U_{e f f}$ functions calibrated with 5\% versus $20 \%$ retrieval precision. Combining all sources of error in quadrature, we find total uncertainties ( $1 \sigma$ ) of 30-79\% for landfill emissions quantified with GHGSat. 

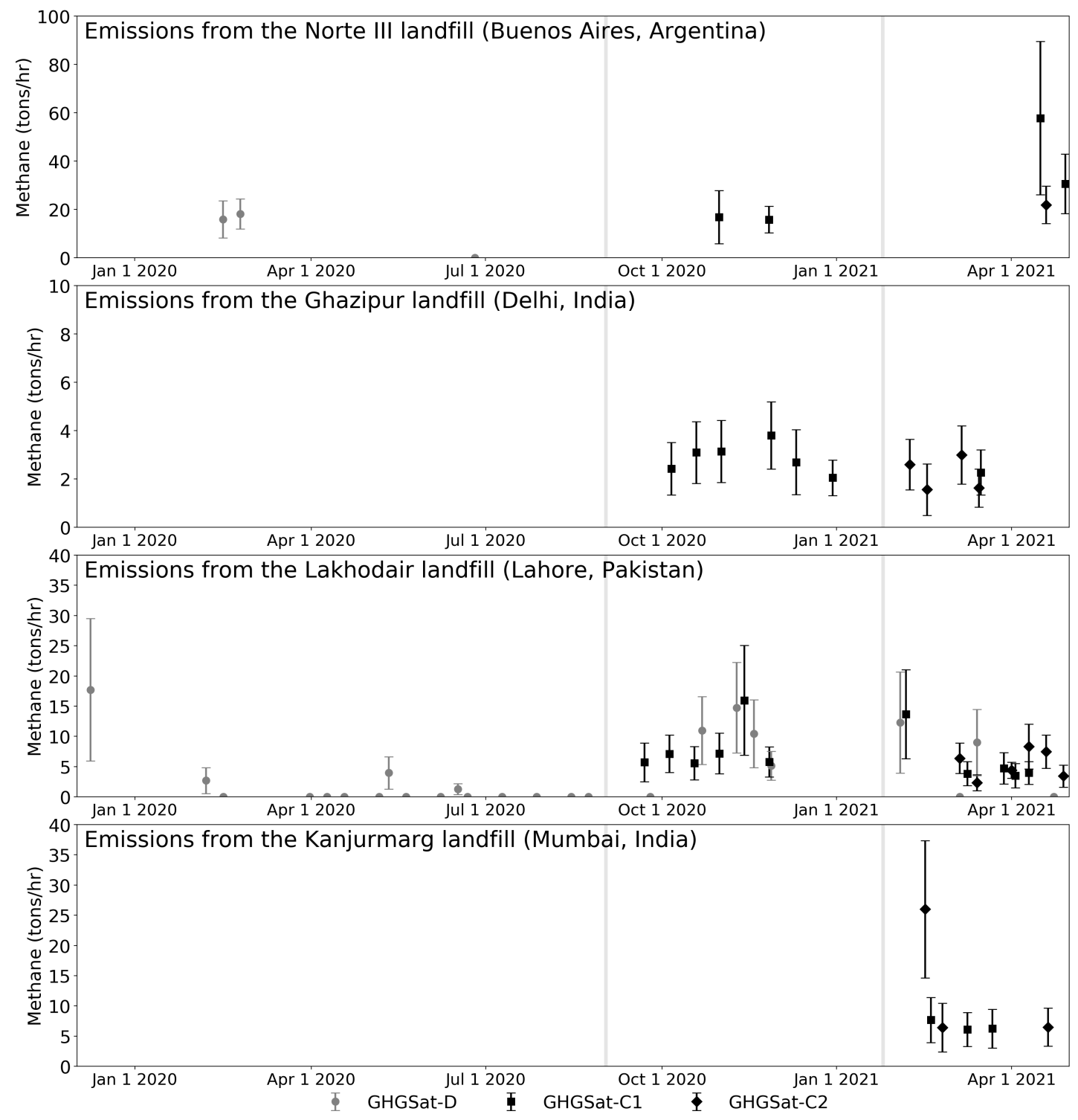

Figure S.7: Time series of GHGSat-D/C1/C2 quantifications for the four landfills. Uncertainties are calculated as described in Supplement 2. Markers at 0 indicate clear scenes without any detected plumes, the grey background lines indicate the launch dates of GHGSat-C1/C2. 

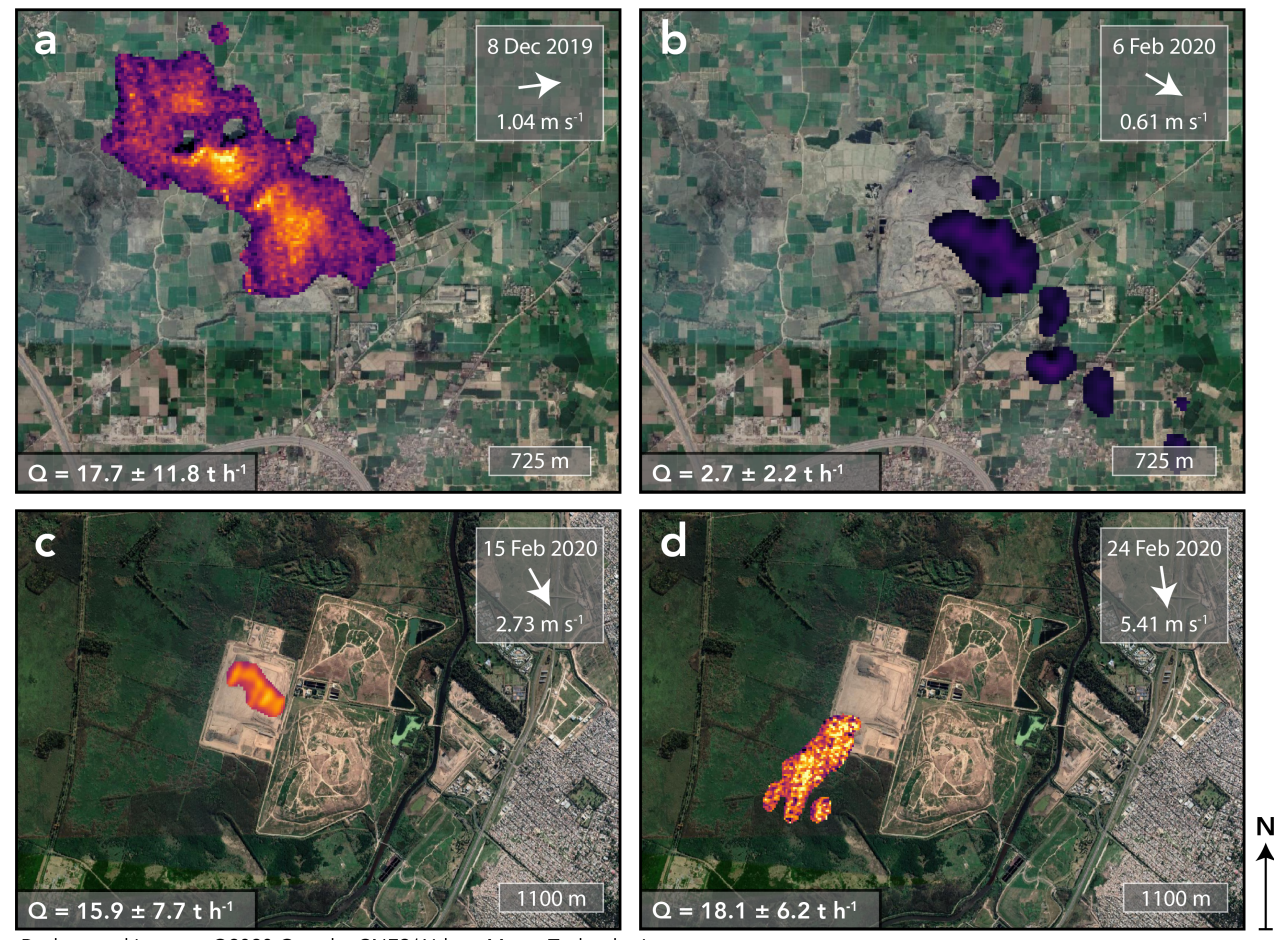

Background imagery @2020 Google, CNES/Airbus, Maxar Technologies

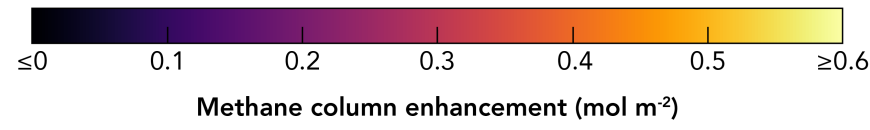

Figure S.8: Methane plumes observed by GHGSat-D from the (a-b) Lakhodair (Lahore, Pakistan) and (c-d) Norte III (Buenos Aires, Argentina) landfills, in December 2019 and February 2020. Concentrations are plotted over high-resolution surface imagery. Wind directions are from GEOS-FP (32) and the emission quantifications are shown in the legend. 


\section{Supplement 3: TROPOMI emission quantification and uncertainty}

We use version 4.1 of the Weather Research and Forecasting (WRF) model (41) to simulate 240 $\times 240 \mathrm{~km}^{2}$ domains around the four landfills at $3 \mathrm{~km}$ resolution from January 1, 2020 to January 1, 2021. The simulations use meteorological fields from the National Centre for Environmental Prediction (NCEP) (51) and initial and 6-hourly boundary conditions at $0.25^{\circ} \times 0.25^{\circ}$ from the Copernicus Atmosphere Monitoring Service (CAMS) (52). Our simulations use the tropical suite of physics options as transport configuration and provide hourly output.

We use bottom-up oil/gas/coal emissions for 2016 from Scarpelli et al. (2020) (38) and remaining anthropogenic emissions are 2015 emissions from EDGAR v5 (39). Wetland emissions (2017) come from WetCHARTs version 1.2.1 (40) mapped to high resolution wetland maps (53).

To estimate mean 2020 emissions, simulation output for that year is sampled using the TROPOMI averaging kernels at the model time step closest to the TROPOMI overpass time. To reduce the impact of possible model errors, we aggregate the TROPOMI observations and their model equivalents to a daily $0.2^{\circ} \times 0.2^{\circ}$ grid and use those aggregated data in a Bayesian inversion to optimize state vector $\widehat{\mathbf{x}}$ :

$$
\widehat{\mathbf{x}}=\mathbf{x}_{\mathbf{A}}+\mathbf{S}_{\mathbf{A}} \mathbf{K}^{T}\left(\mathbf{K S}_{\mathbf{A}} \mathbf{K}^{T}+\mathbf{S}_{\mathbf{O}}\right)^{-1}\left(\mathbf{y}-\mathbf{K x}_{\mathbf{A}}\right)
$$

with posterior error covariance matrix $\widehat{\mathrm{S}}$ :

$$
\widehat{\mathbf{S}}=\left(\mathbf{K}^{T} \mathbf{S}_{\mathbf{O}}{ }^{-1} \mathbf{K}+\mathbf{S}_{\mathbf{A}}{ }^{-1}\right)^{-1}
$$

Where $\mathbf{x}_{\mathbf{A}}$ is the prior state vector; $\mathbf{S}_{\mathbf{A}}$ is the prior error covariance matrix; $\mathbf{K}$ is the Jacobian based on our WRF simulations including the aggregation; $\mathbf{S}_{\mathrm{O}}$ is the observational error 
covariance matrix; and y contains the aggregated TROPOMI observations (with data filtering as described in Supplement 1). The posterior error covariance matrix can be normalized to the posterior error correlation matrix $\widehat{\mathrm{S}_{\text {cor }}}$ by dividing all terms by the square root of the associated diagonal terms.

Our 50-element state vector consists of a scaling factor on the CAMS boundary conditions to prevent bias in CAMS from compromising the inversion results and a $7 \times 7$ grid to scale the bottom-up emissions. When reporting results, the city-level emissions are calculated over $0.8^{\circ} \times 0.8^{\circ}$ boxes centered on the population weighted city centroids.

The prior error covariance matrix is assumed to be diagonal and we assume errors of $50 \%$ for the different emissions and $10 \%$ for the CAMS boundary conditions. The observational error covariance is assumed to be diagonal as well and the error on individual observations is estimated as the standard deviation of the prior model-observation mismatch (17 ppb for Buenos Aires). If $n$ observations fall within one $0.2^{\circ} \times 0.2^{\circ}$ grid cell we apply the central limit theorem $(\sim \sqrt{n})$

To estimate the uncertainty in our results, we generate an ensemble of sensitivity inversions by varying inputs and inversion assumptions. We report the range of these sensitivity inversions as the uncertainty on our emission estimates. The sensitivity inversions are: $(1,2)$ Increasing and decreasing the prior errors by a factor two; $(3,4)$ using WRF model output sampled at the model time steps before and after the mean overpass time; $(5,6)$ performing the optimization with aggregation to $0.15^{\circ} \times 0.15^{\circ}$ and $0.4^{\circ} \times 0.4^{\circ}$ grid cells; (7) offsetting both the latitude and longitude of the aggregation grid by $0.1^{\circ} ;(8)$ using log-normal prior errors on the emissions following Maasakkers et al. (2019); (9) using a 1\% prior error on the CAMS boundary condi- 
tions; (10) only using TROPOMI data with the highest quality flag $(Q A=1)$, (11) using the TROPOMI data without albedo correction, and (12) using the mean observational error for the aggregated observations instead of following the central limit theorem.

Figure S.9 shows the prior and posterior model simulations' mismatches with the TROPOMI observations for 2020 over Buenos Aires. The prior model shows a large-scale underestimate across the model domain due to underestimated CAMS boundary conditions. This underestimate is corrected by scaling up the boundary conditions by $2.9 \%$ in the posterior model (center panel), similar corrections are found for the other cities. The resulting posterior emissions, scaling factors, and the inversion's averaging kernels for the emission grid are shown in Figure S.10. The averaging kernels of the inversion show where the TROPOMI observations add information to the prior emissions. This is mainly the case for the considered urban area, where methane enhancements are seen in the TROPOMI data. Some bias unrelated to local emissions remains in the posterior model - observation mismatch. The resulting city-level emissions are given in Table 1. 

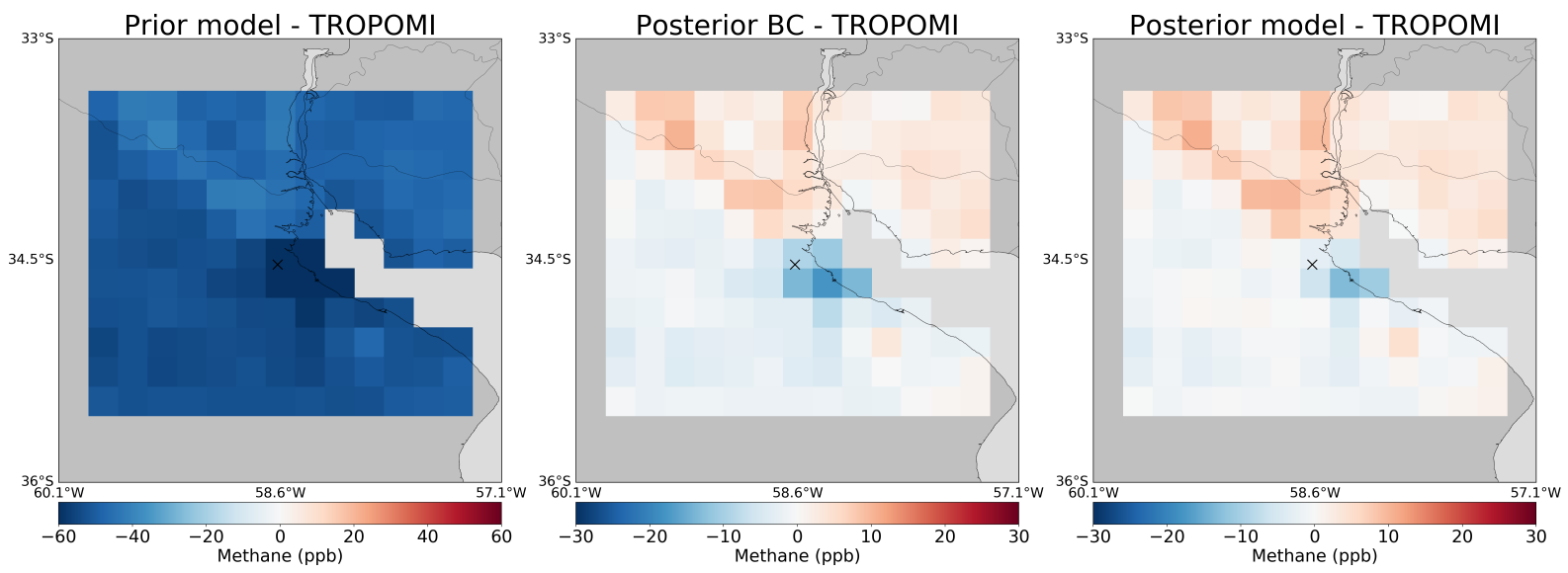

Figure S.9: Model-observation mismatch using the prior (left), correcting the CAMS boundary conditions (center), and posterior (right) models for Buenos Aires compared to 2020 TROPOMI data. Differences are shown on the $0.2^{\circ} \times 0.2^{\circ}$ aggregation grid used in the inversion, only grid cells with at least 20 days of observations are shown. The largest improvement can be seen around the city of Buenos Aires. Different color scales are used in the prior and posterior panels. 

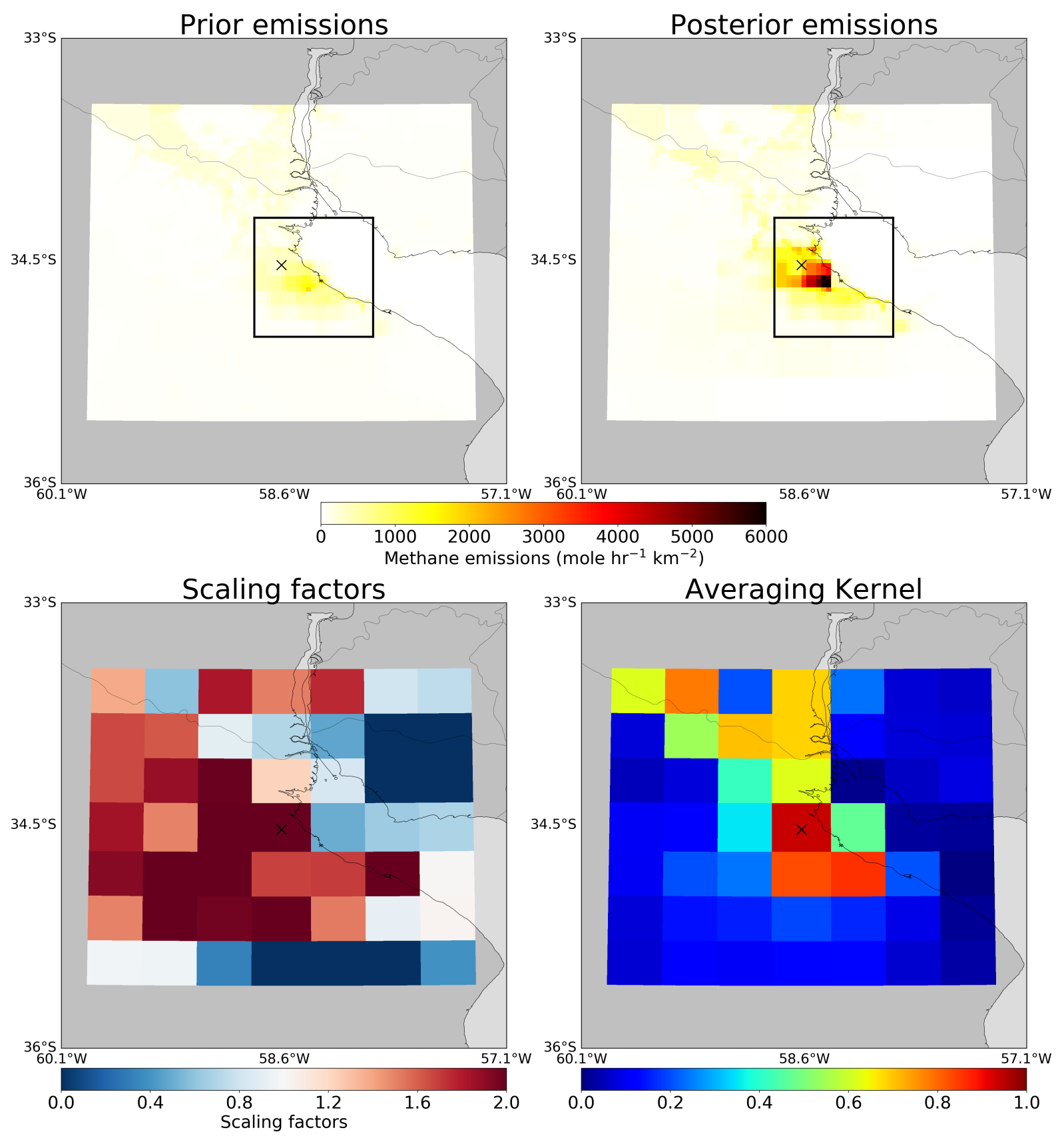

Figure S.10: Prior (a) and posterior (b) emissions from the city-level TROPOMI inversion for Buenos Aires shown at the $3 \times 3 \mathrm{~km}^{2}$ WRF grid, the black box indicates the domain used to estimate urban emissions. Also shown are the resulting scaling factors (c) and the inversion's averaging kernels (d) at the resolution of the inversion. 


\section{Suggested Supplement 4: Map of the Norte III landfill in Buenos Aires}

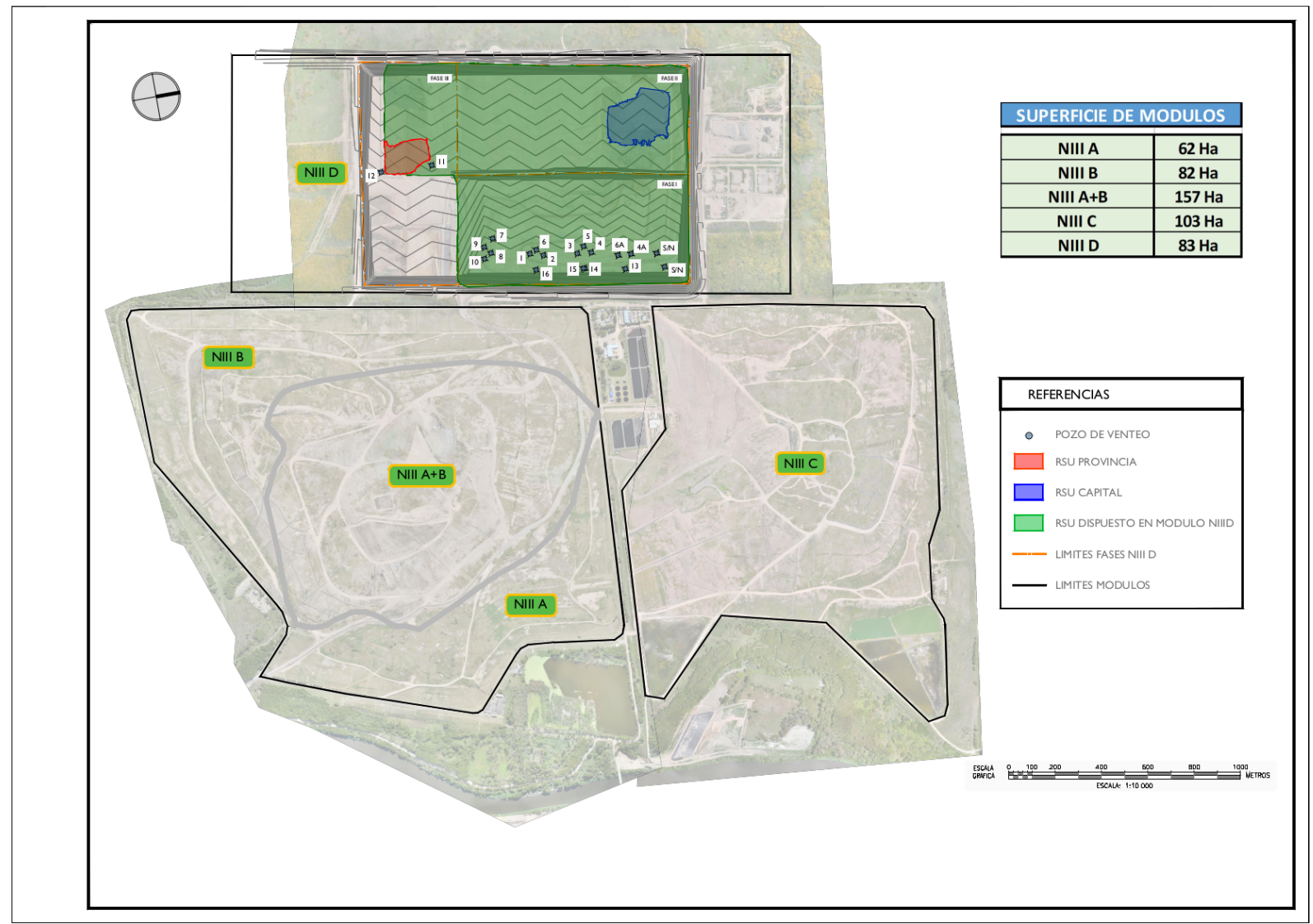

Figure S.11: Overview of the Norte III landfill in Buenos Aires. The active module (D) is located on the western side (top of the figure) of the landfill and includes the provincial (red) and capital (blue) active surfaces. Boreholes are indicated by circles. The inactive modules $\mathrm{A}, \mathrm{B}$, and $\mathrm{C}$ are located on the eastern side of the landfill. 